\title{
Picoeukaryotes of the Micromonas genus: sentinels of a warming ocean
}

\author{
David Demory ${ }^{1,2,3} \cdot$ Anne-Claire Baudoux ${ }^{4}$ Adam Monier $\mathbb{D}^{5} \cdot$ Nathalie Simon ${ }^{4} \cdot$ Christophe Six $^{4} \cdot$ Pei Ge $^{4}$. \\ Fabienne Rigaut-Jalabert ${ }^{6} \cdot{\text { Dominique } \text { Marie }^{4} \cdot \text { Antoine Sciandra }^{2} \cdot \text { Olivier Bernard }^{3} \cdot \text { Sophie Rabouille }}^{2}$
}

Received: 28 August 2017 / Revised: 15 May 2018 / Accepted: 11 June 2018 / Published online: 16 August 2018

(c) International Society for Microbial Ecology 2018

\begin{abstract}
Photosynthetic picoeukaryotesx in the genus Micromonas show among the widest latitudinal distributions on Earth, experiencing large thermal gradients from poles to tropics. Micromonas comprises at least four different species often found in sympatry. While such ubiquity might suggest a wide thermal niche, the temperature response of the different strains is still unexplored, leaving many questions as for their ecological success over such diverse ecosystems. Using combined experiments and theory, we characterize the thermal response of eleven Micromonas strains belonging to four species. We demonstrate that the variety of specific responses to temperature in the Micromonas genus makes this environmental factor an ideal marker to describe its global distribution and diversity. We then propose a diversity model for the genus Micromonas, which proves to be representative of the whole phytoplankton diversity. This prominent primary producer is therefore a sentinel organism of phytoplankton diversity at the global scale. We use the diversity within Micromonas to anticipate the potential impact of global warming on oceanic phytoplankton. We develop a dynamic, adaptive model and run forecast simulations, exploring a range of adaptation time scales, to probe the likely responses to climate change. Results stress how biodiversity erosion depends on the ability of organisms to adapt rapidly to temperature increase.
\end{abstract}

Electronic supplementary material The online version of this article (https://doi.org/10.1038/s41396-018-0248-0) contains supplementary material, which is available to authorized users.

David Demory

david.demory@biosci.gatech.edu

$\triangle$ Olivier Bernard

olivier.bernard@inria.fr

$\triangle$ Sophie Rabouille

srabouille@obs-vlfr.fr

1 School of Biology, Georgia Institute of Technology, Atlanta, GA, USA

2 Sorbonne University, UPMC Univ Paris 06, INSU-CNRS, UMR 7093, Laboratoire Océanographique de Villefranche, 181 Chemin du Lazaret, 06230 Villefranche-sur-mer, France

3 University of Côte d'Azur, INRIA, BIOCORE team, BP93, 06902 Sophia-Antipolis Cedex, France

4 Sorbonne University, UPMC Univ Paris 06, CNRS, UMR 7144, Station Biologique de Roscoff, 29680 Roscoff, France

5 Biosciences, University of Exeter, Exeter, UK

6 Sorbonne University, UPMC Univ Paris 06, CNRS, Fédération de Recherche FR2424, Station Biologique de Roscoff, 29680 Roscoff, France

\section{Introduction}

The Intergovernmental Panel for Climate Change (IPCC) stressed unequivocal warming of the climate system. Their Fifth Report anticipates rises in the global mean surface temperature by the end of $21 \mathrm{st}$ century ranging from $0.3-1.7{ }^{\circ} \mathrm{C}$ (RCP2.6) to $2.6-4.8^{\circ} \mathrm{C}$ (RCP8.5) [1]. Oceans participate in buffering the increasing emissions of greenhouse gases, thus modulating the warming; in addition to the chemical equilibration of gas species between the atmosphere and dissolved phases, phytoplankton is an important contributor of carbon remediation through $\mathrm{CO}_{2}$ sequestration in the ocean [2]. Should dramatic shifts occur in species biodiversity and distribution following temperature increases [3, 4], the resilience of ecosystems could severely be impaired. The likely responses of ecosystems to such rapid temperature changes are at the core of debates, with worrisome consequent impacts on oceanic biogeochemical cycles and feedbacks on the climate system [5].

Phytoplankton live in a thermally fluctuating environment that constrains growth capacity $[3,6,7]$. The temperature growth response of phytoplankton varies widely, both between and within taxa. Phenotypic plasticity 
determines the ability to acclimate to short-term environmental variations while genetic adaptations characterize evolutionary processes under long-term changes. These features will provide, or not, each species with the capacity to survive in a given biotope and to evolve by modifying their thermal niche. Since temperature depends on latitude [8-11], it is therefore a probable driver of niche partition in the oceans, creating large-scale biogeographic patterns [12]. Hence, the structure and diversity of phytoplankton communities could partly reflect observed trends in the global temperature [6, 7].

Temperature-related interspecific distributions have been studied for the whole phytoplankton community [3] but few studies explored intragenus diversity [13, 14]. Micromonas species have emerged as emblematic representative of the eukaryotic pico-phytoplankton communities, thriving in a variety of ecosystems from polar to tropical waters [15-18]. They often dominate phytoplankton in coastal environments [19], where their major contribution to primary production influences the biogeochemical cycles [20]. In the past decade, phylogenetic analyses identified several distinct genetic lineages within Micromonas and have suggested that this genus was composed of cryptic species [21-24]. Four species have now been formally described [25]. Micromonas spp. may co-occur at various latitudes, but were found to occupy different temporal or depth niches within their sympatric ranges [23].

As observed for picocyanobacteria $[13,26]$, the temperature response of such a widely distributed and phylogenetically diverse eukaryote is expected to vary between Micromonas species. The interspecific diversity within the genus Micromonas, the number of characterized strains, and abundant omics data make it a relevant model organism to both explore the impact of temperature on latitudinal distribution and diversity of phytoplankton, and to shed light on the mechanisms that drive phytoplankton thermal responses in the ocean. We therefore studied the thermotolerance and thermal growth response of eleven Micromonas strains in the laboratory under controlled conditions (hereafter referred as experimental strains) and we derived a mathematical model that describes the impact of temperature on growth rate. With this model, we uncover the logic that lies behind the observed distribution of species and their co-occurrence; we also reveal the existence of thermotypes within the genus. We extrapolated the thermal response to a set of 46 additional strains from the Roscoff Culture Collection (hereafter referred as collection strains), observed in various oceanic regions, showing that temperature is the main driver of diversity and distribution in this genus. Then, we developed a predictive model of niche partition to characterize
Micromonas interspecific diversity, which we successfully validated against the Tara Oceans dataset [27], making it a plausible prediction tool. We demonstrated that Micromonas distribution is a relevant and accurate proxy of the whole phytoplankton community distribution. More than a sentinel of the ocean biogeochemistry as previously suggested by Worden and colleagues [28], Micromonas is a probe for global warming. To explore how phytoplankton communities may respond to a future, warmer ocean, we ran the niche partition model under IPCC Sea Surface Temperature (SST) projections, adding an evolutionary model that accounts for the potential adaptation of growth to temperature changes.

\section{Methods}

A graphic abstract of the overall, scientific approach is provided in Supplementary Fig. 1.

\section{Growth measurements and thermal response model}

\section{Culture conditions}

Eleven Micromonas spp. strains were selected from the RCC for the laboratory experiments. We chose strains representative of all the currently known species and according to their isolation site, to consider a range of organisms found along a latitudinal gradient (Supplementary Table 1). Cells were grown in batch cultures in ventilated polystyrene flasks (Nalgene, Rochester, NY, USA) in K-Si medium [29]. Cultures were maintained in temperature-controlled chambers (Aqualytic, Dortmund, Germany) at different temperatures $\left(4,7.5,9.5,12.5,20,25,27.5,30\right.$, and $\left.32.5^{\circ} \mathrm{C}\right)$ for two months (see Supplementary Table 2 for the number of generations) under a $12 \mathrm{~h}: 12 \mathrm{~h}$ light-dark cycle with 100 $\mu \mathrm{mol}$ photons $\mathrm{m}^{-2} \mathrm{~s}^{-1}$ provided by fluorescent tubes (Mazda 18WJr/865).

\section{Growth response curves}

Cell concentration was determined on fresh samples using flow cytometry according to [30]. The maximum cell growth rate $\left(\mu_{\max }\right)$ was calculated as the slope of the linear regression relating cell concentration logarithm $v s$. time observed during the exponential phase of growth. The Cardinal Temperature Model with Inflection (BR model) from [31] was used to estimate the optimal temperature of growth $\left(T_{\text {opt }}\right)$ at which the growth rate is optimal $\left(\mu_{\mathrm{opt}}\right)$, and the minimal and maximal temperatures of growth ( $T_{\min }$ and $T_{\max }$ ) at which $\mu=0$. The growth $\mu(T)$ at 
temperature $T$ is described as follows:

$\mu(T)= \begin{cases}0 & \text { for } T<T_{\min } \\ \mu_{\text {opt }} . \phi(T) & \text { for } T_{\min }<T<T_{\max } . \\ 0 & \text { for } T>T_{\max }\end{cases}$

where

$\phi(T)=\frac{\left(T-T_{\max }\right)\left(T-T_{\min }\right)^{2}}{\left(T_{\text {opt }}-T_{\min }\right)\left[\left(T_{\text {opt }}-T_{\min }\right)\left(T-T_{\text {opt }}\right)-\left(T_{\text {opt }}-T_{\max }\right)\left(T_{\text {opt }}+T_{\min }-2 T\right)\right.}$

\section{Selection of the thermal growth response model}

Number of models exist that represent the response of phytoplankton strains to temperature; we selected the one we believe to be the most relevant for the purpose of the present study. We first short-listed the most appropriate models after the two recent reviews of [32] and [33]. Grimaud and colleagues [32] discussed the strengths and limitations of several thermal response models in regard to four criteria: the fit quality, the easiness of calibration, the biological interpretation of parameters, and the applicability to phytoplankton growth. They convincingly argued that the BR (Eq. (1), [31]) and Eppley-Norberg (Eq. (2), [34]) models presented the overall best performances. Following the analysis from [33], we also considered the Boatman model (Eq. (3), [35]) and we calibrated all three models to our growth measurements (Supplementary Figs. 4 and 5).

$\mu(T)=\left[1-\left(\frac{T-T_{o p t}}{w}\right)^{2}\right] a e^{b T}$ where $w=\left|T_{\max }-T_{\min }\right|$

$\mu(T)=\mu_{\max }\left[\sin \left(\pi \frac{T-T_{\min }}{T_{\max }-T_{\min }}\right)^{a}\right]^{b}$

We then computed an Akaike Information Criterion and a Bayesian Information Criterion (BIC) for each model (Supplementary Table 5) according to the following equations:

$$
\begin{aligned}
& A I C=2 k-2 \ln (M S E) \\
& B I C=-2 \ln (A I C)+k \ln (n)
\end{aligned}
$$

Where $k$ is the number of model parameters to be estimated, MSE the mean square error between measured and predicted growth rates and $n$ the number of data points. These two criteria provide with an estimation of the relative quality of the models tested. Being an increasing function of MSE and $k$, the BIC is a selection criterion between models. The BR model yielded the smallest criteria and, in this regard, represented the best model tested to represent the growth response to temperature in Micromonas, in agreement with the findings of [32] for other phytoplankton species.

\section{Phylogenetic tree reconstruction and evolutionary placements}

\section{Sequence alignment}

18S amplicon sequences from Micromonas RCC strains were aligned to a reference Mamiellophyceae sequence alignment. This reference alignment spans the rDNA operon and was originally used to describe the phylogenetic relationships amongst Mamiellophyceae genera (Marin and Melkonian, 2010). The reference alignment was trimmed to represent only the $18 \mathrm{~S}$ rDNA region; long Micromonas RCC $18 \mathrm{~S}$ amplicons (>1000 nt; $n=35)$ were added to this alignment using MAFFT v7 [36]. The resulting alignment was then edited using the mask from the original alignment annotation [24] and was composed of a total of 2158 sites.

\section{Phylogenetic tree reconstruction}

The edited alignment was used for maximum-likelihood (ML) tree reconstructions. The best ML tree was identified from 100 independent tree reconstructions. All ML reconstructions were run using RAxML v8 [37] with the HKY85 $+\mathrm{G}+\mathrm{I}$ model, which was determined as the best-fit model of nucleotide substitution with jModelTest v2 [38] and by both the Akaike and Bayesian information criteria. Node supports of the resulting phylogenetic tree were determined using 1000 non-parametric bootstrap replicates. Bayesian inferences were conducted using BEAST v2 [39] using the HKY85 + I + G with a log-normal, relaxed molecular clock and default priors. A total of 4 MCMC chains of $10^{6}$ generations were conducted, and a $25 \%$ "burnin" value was applied on the resulting tree set. The iTol web-server [40] was used to generate vector scalable graphic rendering.

\section{Evolutionary placements}

RCC 18S amplicon sequences shorter than 1000 nt $(n=24)$ were placed onto the ML phylogeny using the Evolutionary Placement Algorithm (EPA) implemented in RAxML v8 [41]. Short RCC sequences were aligned with MAFFT v7 against the previously generated updated reference Mamiellophyceae 18S alignment (i.e., composed of reference Mamiellophyceae and long RCC amplicon sequences). The aligned short sequences were then placed onto the reference phylogeny using RAxML in EPA mode with the HKY85 + I + G model. 


\section{Thermal niche partitioning analysis}

\section{Thermal environment dataset}

Using SST from the National Oceanic and Atmospheric Administration's (NOAA), we built a dataset gathering the environmental temperatures at the isolation site of the eleven experimental and 46 Micromonas collection strains referenced in the RCC. At each strain's isolation site, we retrieved the yearly average $\operatorname{SST}\left(\bar{T}_{S}\right)$, minimum SST $\left(T_{S}^{-}\right)$, maximal $\operatorname{SST}\left(T_{S}^{+}\right)$and thermal amplitude $\left(T_{S}^{+}-T_{S}^{-}\right)$ corresponding to a 10-year average (2005 to 2014).

\section{Thermal environment analysis}

To identify possible correlation of isolated strains to temperature, a non-metric dimensional scaling (NMDS) was realized on a Euclidean distance matrix computed on the thermal environment dataset $\left(T_{S}^{-}, \bar{T}_{S}, T_{S}^{+}, T_{S}^{+}-T_{S}^{-}\right)$using the $\mathrm{R}$ package vegan [42]. The stress value is the measure of how well the NMDS configuration represents the dissimilarities and is referred as the Kruskal stress [43].

\section{Relation between strains and environmental temperatures}

Relationships between environmental temperatures $\left(T_{S}^{-}, \bar{T}_{S}\right.$, $T_{S}^{+}, T_{S}^{+}-T_{S}^{-}$), latitude of the isolation site ( $\left.L a t\right)$ and the species cardinal parameters $\left(T_{\min }, T_{\mathrm{opt}}, T_{\max }\right.$, and $\left.\mu_{\max }\right)$ were calculated for the eleven experimental strains that were grown in the laboratory. We tested simple and multiple linear regression models and chose the best relationship according to a high $\mathrm{R}_{\text {adjusted }}^{2}$ and $p$-value $<0.05$. Best relationships were obtained with $\bar{T}_{S}$ and were used to determine cardinal parameters of all other 46 collection strains that were not experimentally tested but referenced in the RCC.

\section{Thermotypes construction}

For each thermotype, we computed 100,000 growth vs. temperature curves through a Monte Carlo procedure with the BR model [31] and cardinal parameters of the $i$-th thermotype randomly taken from the parameter distributions (assuming a gaussian repartition of the parameters in the interval $\left[p^{*}-2 \sigma, p^{*}+2 \sigma\right]$ where $p^{*}$ are the parameters value). In order to ensure a biological coherence in the random samples of the cardinal parameters, the $\mu_{\text {opt }}$ parameter is generated slightly differently. An Eppley model is used to link $\mu_{\mathrm{opt}}$ and $T_{\mathrm{opt}}$ [44]:

$\mu_{\text {opt }}=a \cdot e^{b . T_{o p t}}$

Where parameters $a$ and $b$ are obtained from the best fit with all the strains of the thermotype (Supplementary Table 7).
The values of $\mu_{\mathrm{opt}}$ for a random strain are then directly deduced from random values of $T_{\mathrm{opt}}$ using this model.

Finally, we used the BR model to get the average thermal response and its standard deviation for each thermotype.

The optimal growth response envelope [45] for the whole Micromonas genus was calculated with a BR curve calibrated on a data set consisting in 57 couples $\left(T_{\text {opt }}, \mu_{\text {opt }}\right)$ from the eleven experimental strains and the 46 collection strains. Moreover, the decreasing part of the curve was constrained with 8 couples $(T, \mu(T))$ simulated from the $M$. commoda Warm thermotype model for temperatures equally distributed in the $\left(T_{\mathrm{opt}}, T_{\max }\right)$ interval for this thermotype. The increasing part of the curve was also constrained with eleven couples $(T, \mu(T))$ simulated from the $M$. polaris model for temperatures equally distributed in the $\left(T_{\min }, T_{\mathrm{opt}}\right)$ interval for this species.

\section{Tara Oceans}

\section{Tara Oceans V9 dataset analysis}

Molecular and contextual data from the Tara Oceans project were retrieved from PANGAEA [46]. The Tara Oceans V9-18S dataset [27] is available both at the barcode level (non-redundant sequences) and clustered at the Swarm/ operational taxonomic unit level [47]. Micromonas-like V9-18S barcode sequences were retrieved based on the original taxonomic classification from the Tara Oceans consortium, which was conducted with the Protist Ribosomal Reference database [48] for the protist barcode subset. The resulting 1084 non-redundant barcodes classified as Micromonas-like, and which represented a total of 95755 occurrences across the V9-18S Tara Oceans sampling (334 samples from 47 stations), were then re-classified using a phylogenetic placement procedure. The nonredundant Micromonas-like V9-18S barcodes were aligned against a reference Mamiellophyceae alignment using the same methodology than for the short $18 \mathrm{~S}$ amplicon sequences from the Micromonas RCC strains, as aforementioned. The V9-18S barcode sequences were then placed onto the Mamiellophyceae and RCC reference tree using RAxML EPA with the HKY85 + I + G model. Based on the placement of the Tara Oceans barcode onto the Micromonas reference subtree, the corresponding taxonomic information (thermotype level) was assigned to the environmental barcode.

\section{Thermotypes inside the Tara Oceans V9 dataset}

To explore the impact of temperature on species occurrence, we computed an NMDS on a Bray-Curtis distance matrix calculated from a community matrix of Micromonas species abundance per station (expressed in 
percentage of barcodes) with the R package "vegan" [42]. Results display a cloud of sampling stations from the different oceanic basins, discriminating surface and deep chlorophyll maximum; the closer proximity of stations, in terms of Bray-Curtis distances, expresses their similarities in their $18 \mathrm{~S}$ diversity. We then fitted environmental variables (nutrients, temperature and mixed layer depth) and total chlorophyll $a$ abundance on the ordination space with the vegan function envfit in vegan package [42] with $p$-value based on 999 permutations was used to assess the significance of the fit.

The Micromonas distribution for each thermotype was computed against yearly SST (from NOAA) for each Tara Oceans station. We then computed Loess regressions with polynomial fitting to illustrate the temperature patterns with the R package "ggplot2" [49].

\section{Global temperature response and diversity index}

\section{Global SST dataset}

We used global SST data from the Copernicus Marine Environment Monitoring Service (product: GLOBAL_REP_PHYS_001_013) to calculate monthly averages SST in the period 1993-2012 at the global scale.

\section{Species distribution as a function of temperature}

Cardinal parameters $\left(T_{\min }, T_{\mathrm{opt}}, T_{\max }\right)$ and optimum growth rate $\mu_{\mathrm{opt}}$ for each thermotype $i$ were used to calculate the growth rate $\mu_{i}(T)$ for each temperature $T$ using the BR model [31]. Then, normalized distribution $\mathcal{D}_{i}(T)$ of each thermotype was calculated following the equation: $\mathcal{D}_{i}(T)=$ $\frac{\mu_{i}(T)}{\sum_{i=1}^{n} \mu_{\text {op }, i}}$ for each temperature $T$ of the global ocean surface. Remark that this normalization removes the effect of other factors which also influence net growth at the same location (nutrients, light, predations, etc.).

\section{Diversity index}

To get a diversity index, we computed 10,000 thermal distribution via a Monte Carlo procedure for each species (Supplementary Fig. 13). We then computed an averaged and standard deviation of a Shannon-like based interspecific diversity index within the Micromonas genus according to Eq. (18) (Supplementary Fig. 14) and compared it with a Shannon diversity index based on Tara Oceans V9 dataset thermotypes relative abundance:

$$
H_{\text {TARA }}(s)=\sum_{i=1}^{n} E(s, i) \ln (E(s, i))
$$

Where $E(s, i)$ is the number of barcodes for the Micromonas thermotype $i$ at the station $s$. The Tara Oceans dataset was used along the transect from station 4 to 125 [27]. The spatial distance between stations was calculated as a distance as the crow flies. In addition, we compare the Shannon-like base interspecific diversity index (Eq. (18)) calculated for Micromonas $\left(H_{M}\right)$ to the diversity index calculated by Thomas and colleagues for the phytoplankton $\left(H_{P}\right)$ with a linear regression model $\left(R_{a d j}^{2}=0.95\right.$ and $p$-value $<0.5)$ :

$H_{P}=83.21 H_{M}+65.05$

Then, we used Eq. (8) to quantify the diversity in the same index as the Thomas et al. study [3] (Supplementary Fig. 15).

\section{Cardinal parameters adaptation model}

\section{Cardinal parameters evolution}

We studied the evolution of diversity in a warmer ocean with a dynamical model of the thermal growth response over the period 2001 to 2100. Projections of future, global temperature regimes were obtained from the NOAA GFDL CM2.1 $[50,51]$ driven with the SRES A2 emissions scenario [52]. This dataset spans from 2001 to 2100 and was also used by Thomas and colleagues [3].

First, we computed the evolution of cardinal parameters $T_{c, i}\left(T_{\min }, T_{\mathrm{opt}}\right.$, and $\left.T_{\max }\right)$ for each thermotype $i$ depending on the temperature $T(t, l, L)$ with $t$ the year, $l$ the latitude and $L$ the longitude. The evolution of cardinal parameters follows Eq. (19), which is parameterized by the number of generations $\mathrm{Na}$ required to adapt to a different temperature (Supplementary Fig. 16):

$$
\begin{aligned}
& \frac{d T_{\text {opt }, i}}{d t}=\frac{N_{i}(T(t))}{N a}\left(T_{o p t, i}^{*}(T(t))-T_{o p t, i}(t)\right) \\
& \frac{d T_{\max , i}}{d t}=\frac{N_{i}(T(t))}{N a}\left(T_{\max , i}^{*}(T(t))-T_{\max , i}(t)\right)
\end{aligned}
$$

Where $T_{o p t, i}^{*}$ and $T_{\max , i}^{*}$ are computed from the derivative of the relationships in Table 1 depending on the local temperature $T(t, l, L)$ :

$\frac{d T_{o p t, i}^{*}}{d t}=0.84 \frac{d T(t, l, L)}{d t}$

$\frac{d T_{\max , i}^{*}}{d t}=0.77 \frac{d T(t, l, L)}{d t}$ 
Table 1 Linear relationship between cardinal parameters and environmental parameters (average temperature at the surface of isolation site, $\bar{T}_{S}$, and the latitude, Lat) for the eleven Micromonas experimental strains tested in this study

\begin{tabular}{llll}
\hline $\begin{array}{l}\text { Cardinal } \\
\text { parameter }\end{array}$ & Model & $R^{2}$ adjusted & $p$-value \\
\hline$\mu_{\text {opt }}$ & $\mu_{\text {opt }}=0.03 \bar{T}_{S}+0.47$ & 0.90 & $5.6810^{-6}$ \\
$T_{\max }$ & $T_{\max }=0.77 \bar{T}_{S}+17.73$ & 0.79 & 0.00014 \\
$T_{\text {opt }}$ & $T_{\text {opt }}=0.84 \bar{T}_{S}+10.24$ & 0.79 & 0.00015 \\
$T_{\min }$ & $T_{\min }=-0.76 \mathrm{Lat}-$ & 0.47 & 0.03 \\
& $0.92 \bar{T}_{S}+49.33$ & & \\
\hline
\end{tabular}

The evolutive minimal temperature of growth was computed contingent to the evolution hypothesis:

$T_{\min , i}^{*}=\left\{\begin{array}{l}T_{\min , i}^{i n i}+T_{\max , i}^{*}-T_{\max , i}^{\text {ini }} \\ \frac{d T_{\min , i}}{d t}=\frac{N_{i}(T(t))}{N a}\left(T_{\min , i}^{*}(T(t))-T_{\min , i}(t)\right)\end{array}\right.$

Constant thermal niche

Dynamical model(Eq. 19)

Where $T_{\text {min }, i}^{i n i}$ and $T_{\text {max }, i}^{\text {ini }}$ are the initial value of $T_{\min , i}$ and $T_{\max , i}$ respectively at time $t=2001$ and $T_{\min , i}^{*}$ is computed from the derivative of the relationships in Table 1 depending on the local temperature $T(t, l, L)$ :

$\frac{d T_{\min , i}^{*}}{d t}=-0.92 \frac{d T(t, l, L)}{d t}$

We constrained $T_{\min , i}^{*}$ and $T_{\max , i}^{*}$ by the envelope curve [45] of the Micromonas genus (Fig. 2b) that represents its evolution boundaries.

Second, we calculated $\mu_{\mathrm{opt}, i}$ at $T_{\mathrm{opt}, i}$ with the BR model calibrated with the cardinal parameters of the envelope curve.

Third, we calculated the related growth rate $\mu_{i}(T)$ of each thermotype $i$ depending on its cardinal parameters $T_{c, i}$ at temperature $T(t, l, L)$ following the BR model [31].

Third, we calculated the diversity for the present (2001 to $2010-H_{\text {now }}$ ) and future (2091 to $2100-H_{\text {future }}$ ) periods following the Eq. (18) averaged on 10 years and expressed as the diversity index used by Thomas and colleagues [3] with the Eq. (8).

\section{Diversity erosion}

We performed this cardinal parameter evolution framework for different values of $\mathrm{Na}$, from fast $(\mathrm{Na}<100$ generations) as highlighted by $[53,54]$ to slow $\left(\mathrm{Na}=10^{9}\right)$ adaptation kinetics. This slow time scale corresponds to 2-6 months in the lab, which means a time scale in the range of years in the natural environment (assuming $\mu$ $=0.2$ day $^{-1}$ as a typical growth rate in the sea). For long-term evolution, we refer to a time scale slower than climate change. We call slow evolution an evolution with a typical adaptation kinetics with a millennium, which means $\mathrm{Na}=10^{6}$ generations for an average growth rate of 0.2 day $^{-1}$. We then calculated a diversity erosion index representing the loss of diversity along the latitude gradient with the equation:

$H_{\text {erosion }}(l)=\frac{h_{L}}{L_{\max }} \sum_{L=0}^{L_{\max }}\left(H_{\text {now }}(l, L)-H_{\text {future }}(l, L)\right)$

With $L$ the longitude and $l$ the latitude, $L_{\max }$ the maximal longitude of the dataset $(n=359.7)$ and $h$ the longitude resolution $\left(h_{L}=0.1\right)$.

The averaged latitudinal erosion $\left(\overline{H_{\text {erosion }}}\right)$ per latitude was calculated as follows:

$\overline{H_{\text {erosion }}}=\frac{h_{l}}{n} \sum_{l=l_{\min }}^{l_{\max }}\left(H_{\text {erosion }}(l)\right)$

With $l$ the latitude, $l_{\min }$ and $l_{\max }$ the minimum and maximum latitude of the dataset $\left(l_{\min }=-82\right.$ and $\left.l_{\max }=90\right), h_{l}$ the latitude resolution $\left(h_{l}=0.1\right)$ and $n$ the $H_{\text {erosion }}$ vector's length. A negative erosion signifies a diversity gain.

The tipping point $(p)$ of the $\overline{H_{\text {erosion }}}$ vs. Na curve was calculated as the inflection point following the equation:

$p=\max \left(\frac{d \overline{H_{\text {erosion }}}}{d N a}\right)$

\section{Results and discussion}

\section{Micromonas strains feature distinct physiological responses to temperature}

To estimate the temperature tolerance and growth responses of the four described Micromonas species, we selected three strains of M. commoda, M. bravo and M. pusilla as well as two strains of $M$. polaris. We measured their exponential growth rate after being grown for two months between 4 and $35^{\circ} \mathrm{C}(41.52 \pm 30.61$ generations on average, Supplementary Table 2) depending on the strain origin. To increase the accuracy in the temperature response estimation, the experimental protocols followed the recommendations given in [55, 32] (see Methods). The chosen strains, obtained from the Roscoff Culture Collection (RCC), were originally isolated from contrasted thermal niches of the Atlantic, Pacific and Artic basins (Fig. 1a, Supplementary Fig. 3 and Supplementary Table 1). All showed a typical $[56,31]$ asymmetric growth response to temperature, which we characterized by four cardinal growth parameters: $T_{\min }$ and $T_{\max }$, respectively the minimum and maximum temperatures for growth; $\mu_{\text {opt }}$, the maximum specific growth rate obtained at the optimum temperature $T_{\text {opt }}$ (Fig. 1b). Overall, the Micromonas genus was able to grow over the 
a

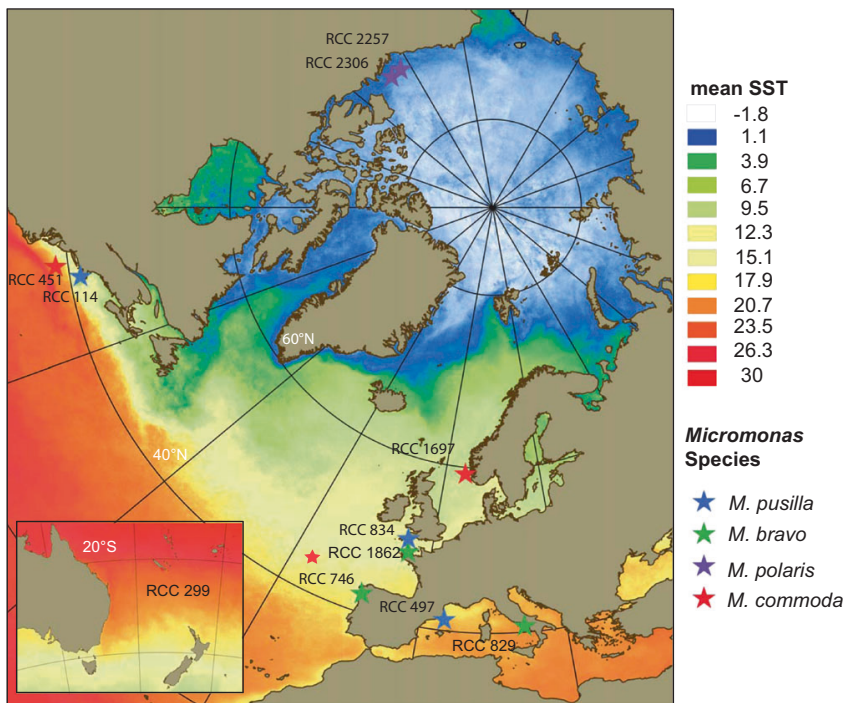

b

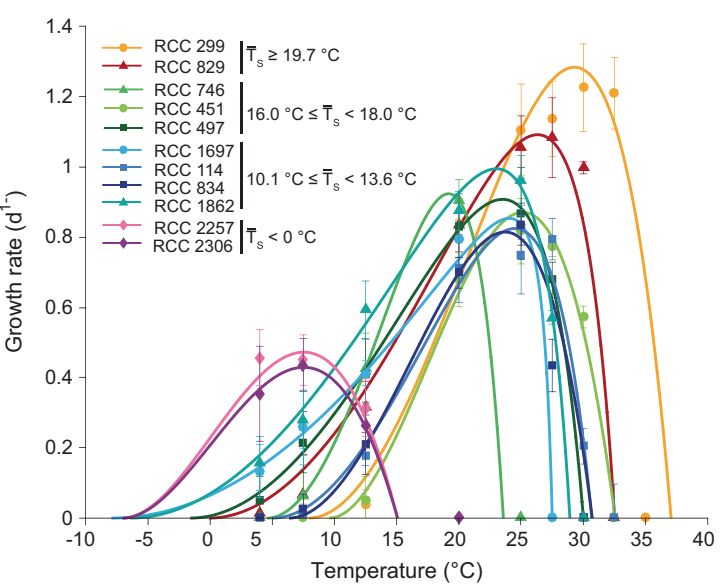

Fig. 1 Micromonas growth response to temperature. a Location of isolation sites of the eleven Micromonas experimental strains used in this study, plotted against yearly average SST for the year 2014 (from
NOAA). b Growth rate vs. temperature curves for strains isolated in environments with different annual average temperature $\left(\bar{T}_{S}\right)$, fitted by the BR model [31]. Error bars are standard deviations $(n \geq 3)$ thermal range tested, but with diverse and specific responses for each strain, depicted by distinct cardinal parameters (Supplementary Table 6). Temperature stimulates enzymatic processes and metabolic rates, but also accelerates cell mortality [57]. In the suboptimal range $\left(T<T_{\text {opt }}\right)$, enzymatic activity increases more than mortality in response to increasing temperatures. At $T_{\mathrm{opt}}$ this balance between metabolic activity and mortality is optimized and yields the highest observed net growth rate. At supra optimal temperatures $\left(T>T_{\mathrm{opt}}\right)$, the denaturation of key metabolic enzymes, like rubisco [58] and the thermolability of Photosystem II [59] are exacerbated, along with an increase of the membrane damages [60]; as a consequence, the net growth rate sharply decreases with temperature up to the maximal growth temperature the strain can withstand ( $T_{\max }$ at which $\mu$ is null).

Several patterns appeared when comparing the growth response to the annual average SST $\left(\bar{T}_{S}\right)$ at the site where each strain was isolated. Strains isolated in locations where $\bar{T}_{S}$ was above $19.7^{\circ} \mathrm{C}$ (RCC 299 and RCC 829) were able to grow up to high temperatures $\left(T_{\max }=32.6 \pm 0.02\right.$ and $37.0 \pm 0.12{ }^{\circ} \mathrm{C}$, respectively); they showed a high $\mu_{\text {opt }}(1.1 \pm$ 0.05 to $1.3 \pm 0.07 \mathrm{~d}^{-1}$, respectively) at an elevated optimum $T_{\text {opt }}$ temperature ( $26.3 \pm 1.01$ to $29.3 \pm 1.2^{\circ} \mathrm{C}$, respectively). Strains isolated in regions where the average SST fluctuates between 16.0 and $18.0^{\circ} \mathrm{C}$ presented a lower optimal growth rate $\left(0.9 \pm 0.03 \mathrm{~d}^{-1}\right)$ at $\left.T_{\mathrm{opt}}=22.6 \pm 3.08^{\circ} \mathrm{C}\right)$ and maintained positive growth from $4.2 \pm 5.6$ to $28.7 \pm 4.63{ }^{\circ} \mathrm{C}$. In strains isolated at sites with an average temperature between 10.1 and $13.6^{\circ}, \mu_{\text {opt }}$ still reached $0.87 \pm 0.08 \mathrm{~d}^{-1}$ at $T_{\text {opt }}=23.8 \pm$ $0.62{ }^{\circ} \mathrm{C}$ and cells demonstrated an ability to grow over a very wide temperature range (from $-0.7 \pm 7.46$ to $29.4 \pm$ $1.55^{\circ} \mathrm{C}$ ). Last, Arctic strains (RCC2306 and RCC2257) revealed both the narrowest growth temperature range $\left(-7.0 \pm 0^{\circ}\right.$ to $\left.15.1 \pm 0{ }^{\circ} \mathrm{C}\right)$ and lowest growth rates $(0.45 \pm$ $\left.0.03 \mathrm{~d}^{-1}\right)$ at $7.5 \pm 0{ }^{\circ} \mathrm{C}$.

In Summary, the four formerly described Micromonas species exhibited specific temperature tolerance and growth optima in vitro and their according response parameters were related to the thermal environment from which the strains were isolated. Model parameters $T_{\min }$, and to a lesser extent $T_{\max }$, are difficult to accurately estimate [31]. Since measurements for temperatures close to $T_{\min }$ (but slightly higher) and close to $T_{\max }$ (but slightly lower) are generally rare, they must be extrapolated from a mathematical model. These parameters also bracket the thermal niche, i.e., the breadth of the thermal response. For instance, it appears that Arctic strains showed a much narrower niche: they were more stenotherm compared to the other strains.

\section{The Micromonas genus includes six thermotypes: evidence from the most recent phylogeny}

The phylogenetic analysis of the 57 Micromonas 18 S DNA sequences from the eleven experimental and 46 collection strains highlighted the existence of six distinct phylogenetic groups (see Methods and Supplementary Fig. 2). To identify whether they were associated with specific thermal conditions in the ocean, we analyzed available data of average SST in areas where Micromonas spp. were sampled. We computed a non-metric dimensional scaling (NMDS) of the thermal environment dataset (see Methods). The significant ordination (stress $=0.004$ ) identified six different distributions in the thermal environment, from 

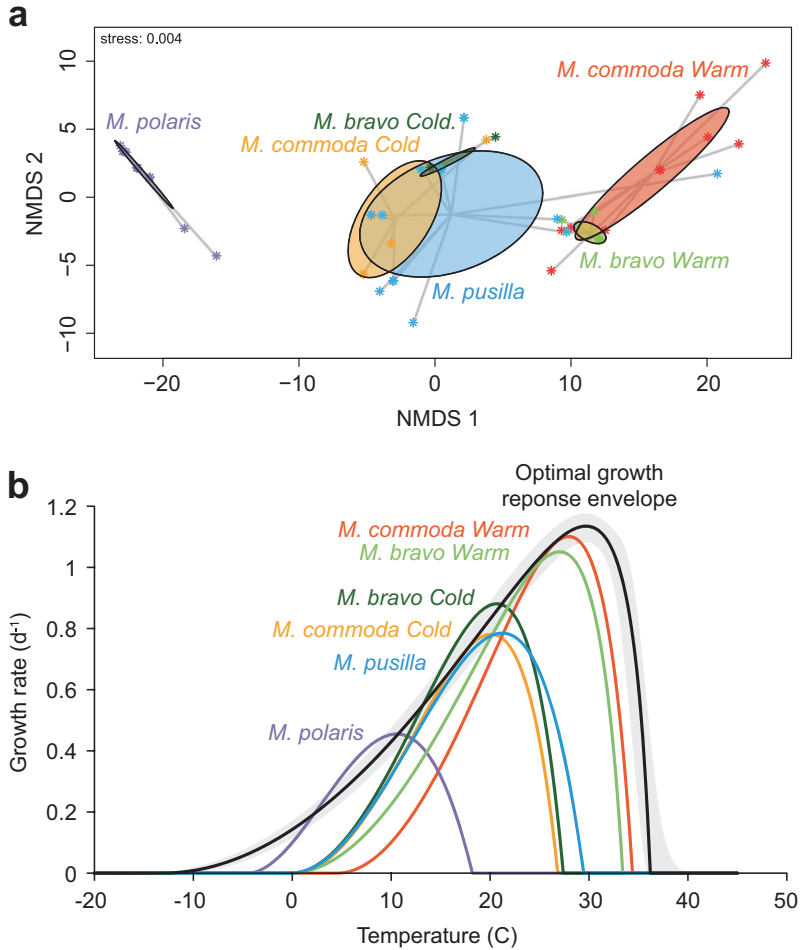

Fig. 2 Original thermal environments and growth response to temperature for Micromonas species. a Two-dimensional ordination space derived from a non-metric multidimensional scaling (NMDS) procedure displaying the thermal dissimilarities site $\left(T_{S}^{-}, \bar{T}_{S}, T_{S}^{+}\right.$and $T_{S}^{+}-T_{S}^{-}$) between the original isolation sites of the eleven experimental and 46 Micromonas collection strains. The stress value (goodness-of-fit of the NMDS) is inferior to 0.05, indicating high dimensional relationships among samples. b Average growth response to temperature for each phylogenetic group computed from 100,000 possible response curves simulated within the ranges observed in each phylogenetic group. The black line represents the overall, optimal growth response envelope [45] of Micromonas computed as $\mu_{\mathrm{opt}}$ vs. $T_{\mathrm{opt}}$, where $\mu_{\mathrm{opt}}$ and $T_{\mathrm{opt}}$ are given by the average response of each thermotype. The grey shaded area is the standard deviation around $\mu_{\mathrm{opt}}$

warmer, low latitudes to colder, high latitudes, that showed a good match with the phylogenetic tree (Fig. 2a and Supplementary Fig. 3), demonstrating that the thermal niche of Micromonas was related to its phylogenetic affiliation. M. polaris and M. pusilla strains occupied respectively a narrow and wide thermal niche while $M$. bravo and M. commoda each included two distinct groups. One isolated from a warmer (lower latitude; warm group) and one isolated in a colder (higher latitudes; cold group) environment (Fig. 2a and Supplementary Fig. 3).

There are few examples in the literature of latitudinal segregation within eukaryotic phytoplankton genera [61, 62]. For example, the global distribution of Ostreococus clades, a picoeukaryote close to Micromonas is related to temperature but first seems to discriminate rather coastal, high-light adapted clades from more oceanic, low-light adapted clades
[63]. In agreement with the hypothesis of Foulon et al. [23], our experimental and phylogenetic results showed that a niche segregation within Micromonas did occur that is consequent to thermal, group-specificities and which compels with the recently identified, four known species. The present analysis further revealed the existence of two thermotypes within both $M$. commoda and $M$. bravo species, making a total of six distinct Micromonas thermotypes.

\section{Establishing a thermal response model for Micromonas thermotypes}

To obtain a better appraisal of the thermal response of strains, we looked for possible correlations between cardinal growth parameters and environmental features where strains had been isolated. Among the tested descriptors of the SST dynamics, the average surface temperature at the isolation site $\left(\bar{T}_{S}\right)$ best correlated with the cardinal temperature. For $T_{\min }$, the latitude was also included in the regression (Table 1 and Supplementary Fig. 6a). The optimal growth rate $\left(\mu_{\mathrm{opt}}\right)$ increased with $\bar{T}_{S}$, following the Eppley's hypothesis of a faster growth rate at warmer temperatures [44]. The maximal growth temperature $\left(T_{\max }\right)$ and the optimal growth temperature $\left(T_{\text {opt }}\right)$ were also both positively correlated with $\bar{T}_{S}$, suggesting that environmental temperature featured the upper tolerance window of strains. The minimal temperature of growth $\left(T_{\min }\right)$ had the lowest correlation with the environmental temperature (Supplementary Fig. 6b), as also reported by [64] for different phytoplankton species. We found that the minimal growth temperature

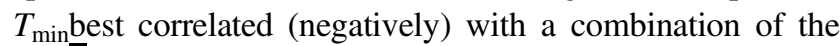
yearly average temperature $\bar{T}_{S}$ and latitude (Lat, Supplementary Fig. 7). In the end, the growth response $\left(\mu_{\mathrm{opt}}, T_{\min }\right.$, $T_{\mathrm{opt}}$, and $T_{\max }$ ) of cultured strains can thus accurately be predicted from the thermal environments $\left(\bar{T}_{S}\right)$ and latitude from which they were isolated, using the relations defined in Table 1. Last, statistically significant correlations were also found between cardinal parameters (Supplementary Fig. 8). In particular, the optimal temperature of growth $\left(T_{\mathrm{opt}}\right)$ linearly correlated with the maximal temperature of growth $\left(T_{\max }\right)$ by a factor close to 1 , as previously highlighted for a wide range of bacterial species [65].

The relationships between cardinal growth parameters and environmental temperatures deduced from the culture experiments (Table 1) were used to extrapolate the cardinal parameters of 46 additional Micromonas collection strains, using the latitude and average annual temperature of their isolation site (Table 1 and Supplementary Table 8). This data set confirmed a segregation of the four species into six different thermotypes. To deduce a representative thermal response for each thermotype, we randomly chose 100,000 values within the confidence interval of the cardinal parameters of each group and ran Monte Carlo simulations of 
a

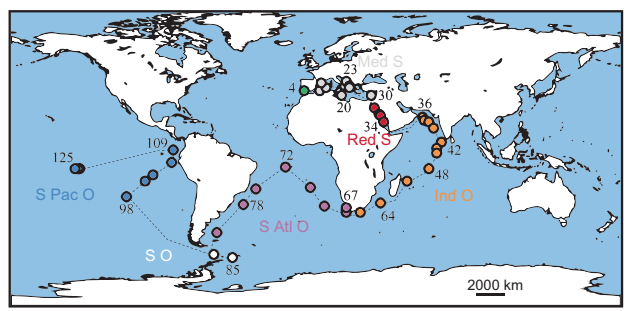

b

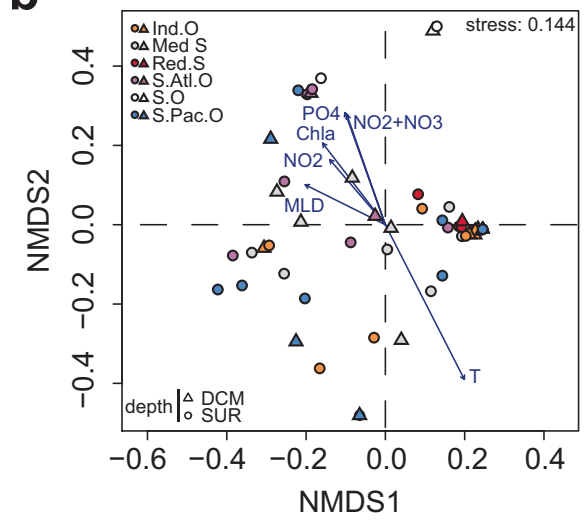

C
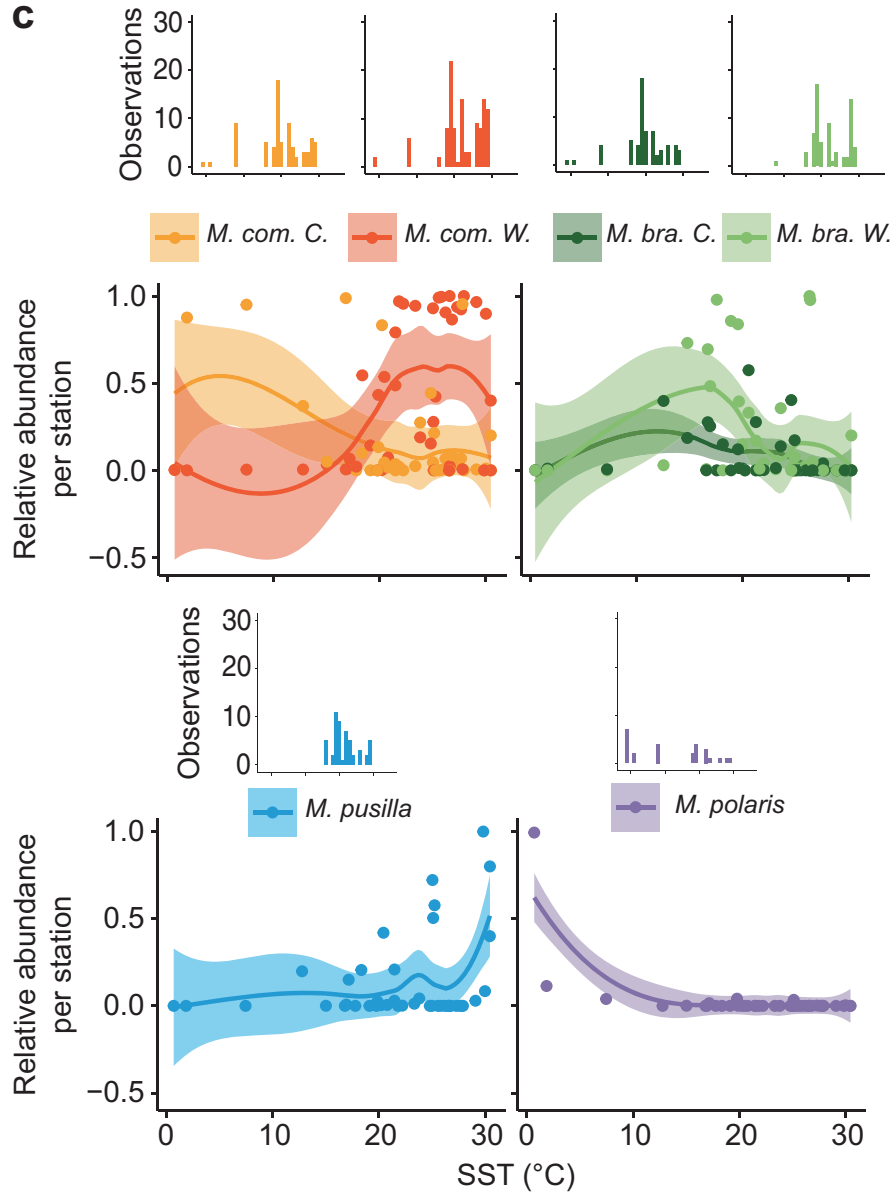

Fig. 3 Micromonas thermotypes relative abundance patterns as estimated from the 18S rRNA V9 region during the Tara Oceans cruise. a Map of the Tara Oceans transect (dashed black line)showing station for which $18 \mathrm{~S}$ rRNA V9 region data were available from Vargas et al. [27]: Mediterranean Sea (Med S), Red Sea (Red S), Indian Ocean (Ind O), South Pacific Ocean (S Pac O), Southern Ocean (S O) and South Atlantic Ocean (S Atl O). b Two-dimensional ordination space derived from an NMDS analysis displaying Bray-Curtis distance between the Micromonas species

the related thermal responses (see Methods). The Bernard and Rémond (BR) model was then fitted to each bundle of simulated responses [31] to obtain the average thermal response curve representative of each thermotype (Fig. 2b and Supplementary Figs. 9-11). Last, we calibrated the envelope curve, inspired from [45], on the Micromonas genus, by fitting the BR model [31] to the set of $\left(T_{\mathrm{opt}}, \mu_{\mathrm{opt}}\right)$ obtained for each thermotype (see Methods and Fig. 2b).

With the narrowest thermal niche $\left(23.04 \pm 2.42{ }^{\circ} \mathrm{C}\right)$, M. polaris was the most stenotherm species. M. commoda cold and M. bravo cold showed very similar responses at colder temperatures but discriminated in regard to the optimum growth rate and maximum temperature. Their thermal niche of $25.42 \pm 3.75$ and $27.10 \pm 0.91{ }^{\circ} \mathrm{C}$, respectively, was representative of cold-temperate environments. Contrary to the cold species, and although they both live in warmer biotopes, the warm thermotype of species

assemblages of the Tara Oceans stations, fitted by significant environmental variable $(p$-value $<0.05)$. The stress value (goodness-of-fit of the NMDS) is 0.15 , indicating fair dimensional relationships among samples. c Relative abundance of the 6 thermotypes per station, plotted according to yearly SST at station coordinates: data (circles) and polynomial regression (solid line) fitted with the $95 \%$ confidence interval (shaded area). Number of observations for the six thermotypes are represented in histograms, plotted according to yearly SST at station coordinates

M. commoda and $M$. bravo showed very distinct thermal niches $\left(34.00 \pm 1.19\right.$ and $26.02 \pm 5.11^{\circ} \mathrm{C}$, respectively). Last, M. pusilla was found in both cold-and warm-temperate areas and showed an intermediate thermal response compared to the other Micromonas species, with a thermal niche of $28.85 \pm 5.32{ }^{\circ} \mathrm{C}$. With the most variable response to temperature, $M$. pusilla did not seem to speciate into different thermotypes; yet it clearly differentiated from other groups and would be the most eurytherm.

\section{Tara Oceans dataset validates the global segregation of thermotypes}

To validate our hypothesis that temperature is a key factor that greatly influences Micromonas biogeography over a yearly period, we retrieved the $18 \mathrm{~S}$ V9 metabarcodes dataset obtained in the frame of Tara Oceans [27] (Fig. 3). 
Read abundance data assigned to each of the Micromonas thermotypes were identified across 47 stations, spanning 6 marine regions with different thermal environments: Mediterranean Sea, Red Sea, Indian Ocean, South Atlantic Ocean, Southern Ocean and South Pacific Ocean (Fig. 3a). Using an NMDS ordination method, we first compared the relative abundance of each Micromonas thermotype at sampling stations (see Methods and Fig. 3b) to the physicochemical environmental conditions observed along the Tara Oceans circumnavigation. The presence of Micromonas species was better explained by temperature $\left(R^{2}=0.48, p\right.$-value $\left.<0.001\right)$ than by nutrient availability, mixing, or geographical location. To a lower extent, nutrients $\left(\mathrm{NO}_{2}+\mathrm{NO}_{3}, \mathrm{PO}_{4}\right.$ and $\mathrm{NO}_{2} ; R^{2}<0.23, p$-value $\left.<0.032\right)$, Chla concentration $\left(R^{2}=\right.$ $0.1710, p$-value $=0.003$ ) and mixed layer depth (MLD; $R^{2}=0.13, p$-value $\left.=0.03\right)$ also explained significantly the Micromonas assemblages along the transect. Temperature is thus the strongest descriptor of the change in diversity between Tara Oceans stations.

We then compared the relative abundance of thermotypes at all stations in relation to yearly SST (Fig. 3c). A very clear thermal separation appeared between the two $M$. commoda thermotypes, further supporting our identification of two distinct thermotypes. M. commoda cold was most abundant in waters with temperature below $20^{\circ} \mathrm{C}$ and rarely found beyond $25^{\circ} \mathrm{C}$, while $M$. commoda warm mostly occurred between 25 and $30^{\circ} \mathrm{C}$ and was completely absent at stations where temperatures were below $15^{\circ} \mathrm{C}$. Species $M$. bravo was less often observed than M. commoda and showed overlapping distributions of its two warm and cold thermotypes, which we believe was due to the large thermal niche of the warm thermotype spreading over that of the more restrained, cold thermotype (Fig. 2). A nondistinct distribution (Fig. 3c) in the Tara Oceans data could also suggest that the evolution of the two $M$. bravo thermotypes was more recent. Species M. polaris was observed only at stations with $\mathrm{T}<10^{\circ} \mathrm{C}$ with highest abundances near $0{ }^{\circ} \mathrm{C}$, validating the psychrophilic characteristics of this thermotype. Species $M$. pusilla was only found at a few stations compared to $M$. commoda and $M$. bravo; it was observed from 12 to $30^{\circ} \mathrm{C}$ with a maximum abundance above $25^{\circ} \mathrm{C}$. This distribution may well be related to the fact that its thermal response is close to the barycenter of the whole Micromonas thermal response (average parameters: $\overline{T_{o p t}}=21.26, \overline{\mu_{o p t}}=0.84$ and $\left.\overline{\left(T_{\max }-T_{\min }\right)}=28.34\right)$. The reported occurrences of this species at low concentrations all around the globe $[23,66]$ could support the idea that it plays a "seed bank" role, acting as a dormancy stage of Micromonas compared to other species [67]. Interestingly, Foulon et al. [23] also suggested a possible niche partition over depth, along a light gradient that may explain the low concentration of $M$. pusilla in the Tara Oceans dataset. In the end, temperature is a sufficient parameter to describe the latitudinal segregation of Micromonas between Tara Oceans stations. The current typology of Tara Oceans (they mainly are open ocean areas), does not allow to fully assess a possible effect of nutrients [68].

\section{Influence of temperature on the intragenus diversity of Micromonas assemblages}

To further understand the thermal niche partition of Micromonas at the global scale, we proposed a simple index to relate Micromonas intragenus diversity to the global average SST (Fig. 4 and Supplementary Fig. 12). We computed an interspecific Micromonas diversity index (Shannon derived/ based) from the growth response of a given thermotype $i$ to a considered local temperature $T$ according to the equation:

$$
H(T)=\sum_{i=1}^{n} \mathcal{D}_{i} \ln \left(\mathcal{D}_{i}\right) \text { with } \mathcal{D}_{i}=\frac{\mu_{i}(T)}{\sum_{i=1}^{n} \mu_{\text {opt }, i}}
$$

Where $\mathcal{D}_{i}$ is the distribution index, $\mu_{i}(T)$ is the growth rate at the temperature $T$, and $\mu_{\mathrm{opt}, i}$ is the optimal growth for the thermotype $i$. We compared $H(T)$ to a Shannon-like index for the Micromonas genus at each Tara Oceans sampling station using the proportion of each Micromonas thermotype OTU in the total counted Micromonas OTU and the local SST annual average (Fig. 4a, b). Based on the calculated diversity index $H(T)$, we were able to qualitatively predict the Micromonas intragenus diversity estimated from the Tara Oceans V9-18S dataset (Spearman test: $\rho=0.417, p$-value $=0.0035$ ), thereby validating our theoretical developments. The diversity index followed a fluctuating trend through the cruise path characterized by different thermal environments (Fig. 3a).

When running the Micromonas diversity model at the global scale (Fig. 4c and Supplementary Fig. 13), the predicted diversity was minimal at the poles (Lat $>60^{\circ} \mathrm{N}$ and $>50^{\circ} \mathrm{S}$ ) and at the equator (between $20^{\circ} \mathrm{N}$ and $20^{\circ} \mathrm{S}$ ), especially in the Indian Ocean and the Pacific Ocean (Fig. 4c). Maximum diversity levels were found from 20 to $60^{\circ} \mathrm{N}$ and from 20 to $40^{\circ} \mathrm{S}$. We used the relationship between the phytoplankton diversity as calculated by Thomas and colleagues [3] and our Micromonas diversity to normalize our diversity index within Thomas's scale (see Methods). Our simulated global Micromonas diversity was point by point compared to the whole phytoplankton potential diversity calculated by Thomas and collaborators [3] (Fig. 4d). We found a very strong relationship between the two diversity patterns $\left(\mathrm{R}_{a d j}^{2}=0.97, p\right.$-value $<0.05$; see Methods and Supplementary Fig. 15). This result strongly suggests that the diversity between Micromonas thermotypes, at mesoscale and on a yearly basis, is representative of the whole phytoplankton community. It likely explains the overall success of the genus to colonize very contrasted 

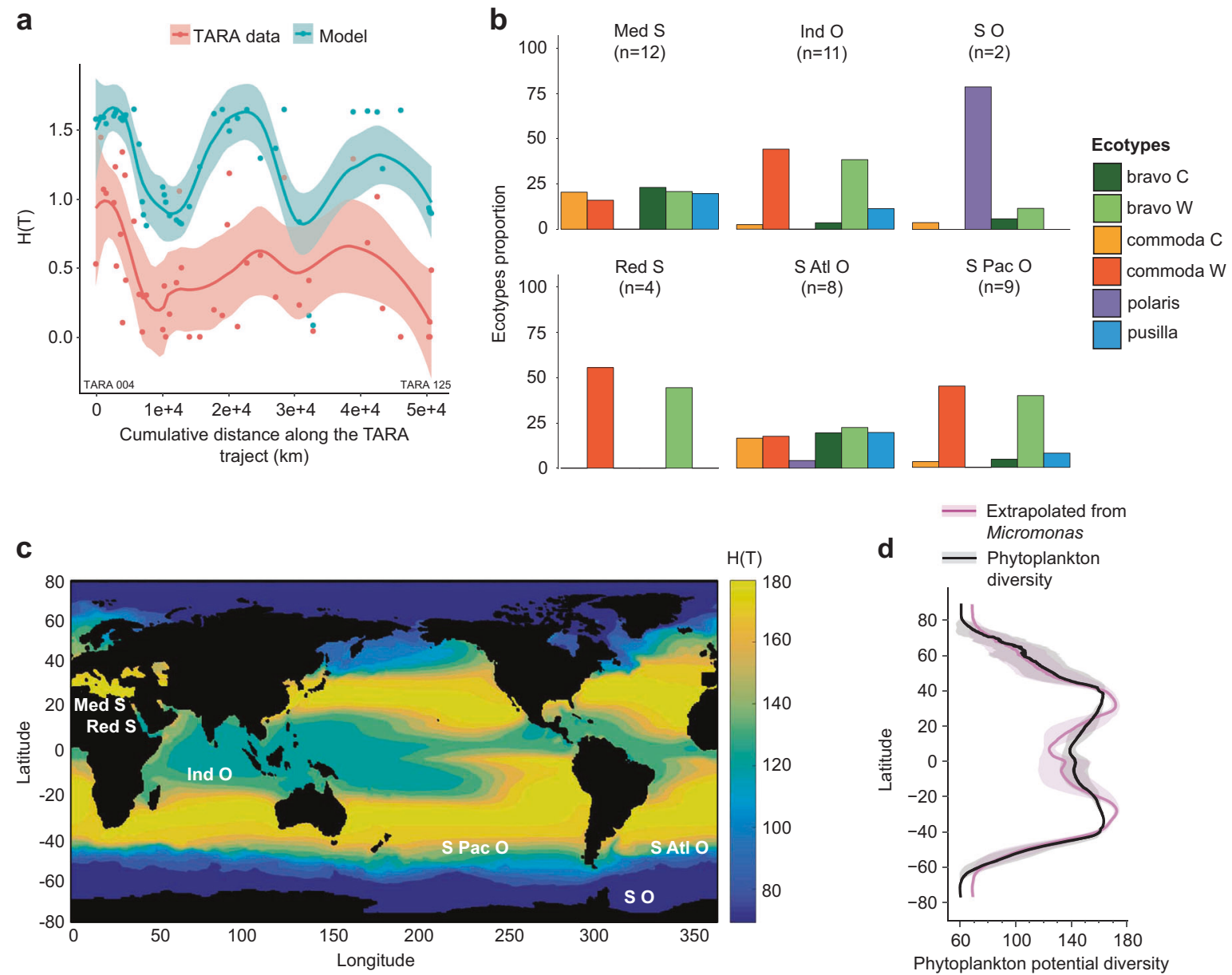

Fig. 4 Estimated and predicted interspecific diversity within the Micromonas genus in the global ocean. a Estimated and predicted interspecific diversity within the Micromonas genus along the Tara Oceans transect as estimated from the Micromonas OTUs read abundances (blue circles) and as predicted from our diversity model (red circles), fitted by a polynomial regression with a $95 \%$ confidence interval. b Thermotypes proportions (\%) from Tara Oceans dataset for different oceanic regions: Mediterranean Sea (Med S), Red Sea (Red

biotopes [19, 23]. Micromonas could thus serve as a relevant marker of the biodiversity of phytoplankton communities. The term "sentinel", originally proposed by [28] to depict the role of Micromonas on ocean biogeochemistry is all the more relevant considering this genus reflects the pattern of the whole phytoplanktonic community and can help to better anticipate the impact of ocean warming.

\section{Diversity evolution in a warmer ocean: a matter of the adaptation time scale}

To explore the impact of future temperature changes on phytoplankton diversity, we investigated its evolution using SST projections over the period 2001-2100. To account for the adaptation capability [4, 69], we proposed a very simple adaptive model. This model assumes that the evolution time scale is related to the local doubling
S), Indian Ocean (Ind O), South Pacific Ocean (S Pac O), Southern Ocean (S O) and South Atlantic Ocean (S Atl O). c Predicted Shannon diversity index $(\mathrm{H})$ calculated with the Eq. (18) using annual averages SST (Copernicus Marine Environment Monitoring Service, 1993 to 2012 satellite data). d Comparison of the latitudinal average diversity for all phytoplankton (from Thomas et al. [3]. black line) with that estimated by our Micromonas model. Shaded area represents the standard deviation from the mean along latitudes

time $\frac{\ln (2)}{\mu_{i}(T)}$ of each thermotype $i$. Adaptation is thus faster for the warm thermotypes in warm environments. The adaptation dynamics describes the evolution of the cardinal temperatures $\left(T_{\min }, T_{\mathrm{opt}}\right.$, and $\left.T_{\max }\right)$ from their present value to their value at the end of the century. The evolution rate is estimated according to the characteristic number of generations $\mathrm{Na}$ required to adapt to a different temperature, i.e., to shift each cardinal (i.e. represented by the character "c") temperature $T_{c}$ to its asymptotic value $T_{c}^{*}$, defined as the evolutionary equilibrium given the changes of the surface temperature $T$ at each time step (Supplementary Fig. 16). The evolution dynamics of each cardinal parameter $T_{c, i}$ is described by a simple first order equation:

$$
\frac{d T_{c, i}}{d t}=\frac{N_{i}(T(t))}{N a}\left(T_{c, i}^{*}(T(t))-T_{c, i}(t)\right)
$$


Where $N_{i}(T(t))=\frac{\mu_{i}\left(T(t), T_{c, i}\right)}{\ln (2)}$, with $\mu_{i}\left(T(t), T_{c, i}(t)\right)$ the growth rate at the temperature $T$, calculated using the set of cardinal parameters $T_{c, i}$ for the thermotype $i$.

We ran this model for different $\mathrm{Na}$, from fast adaptation scales $(\mathrm{Na}<100$ generations) to slow adaptation scales $(\mathrm{Na}=$ $10^{6}$ generations) and calculated the evolution of thermotypes diversity between the present period (2001-2010) and future period (2091-2100, Fig. 5). We considered two realistic evolution hypotheses to describe the dichotomy between specialist and generalist species: the Specialist-generalist hypothesis with constant thermal niche width (Fig. 5a, b) and the Specialist-generalist hypothesis with dynamical thermal niche [70] (Fig. 5c, d-see Methods). Over the 21st century, SST will globally increase by $2-3{ }^{\circ} \mathrm{C}$ over the whole ocean surface and up to $5^{\circ} \mathrm{C}$ around $45^{\circ} \mathrm{N}$, with the exception of the highest latitudes, which may see a slight decrease in their average temperature (Supplementary Fig. 17).

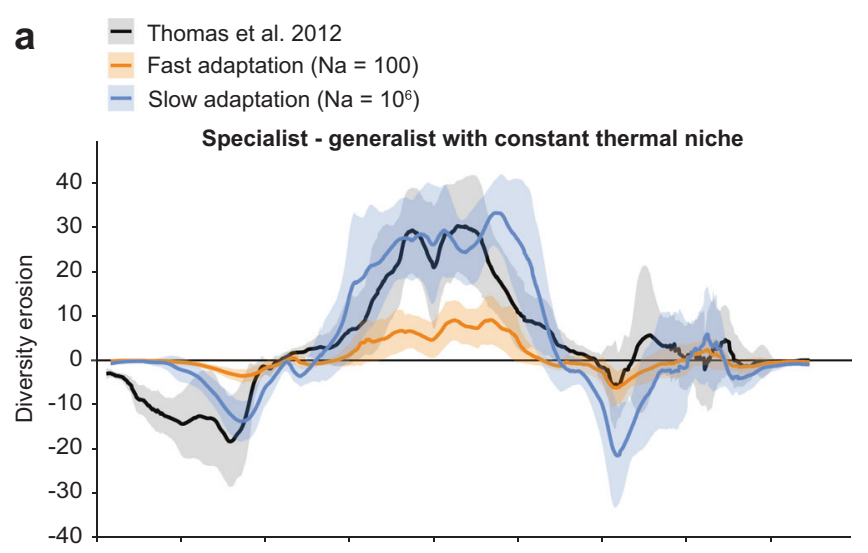

C

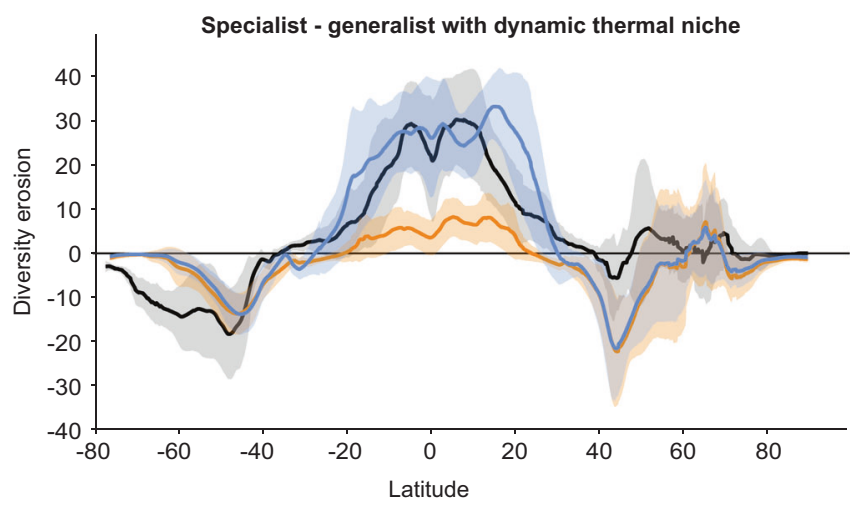

Similar erosion patterns were found for both specialistgeneralist hypotheses that showed diversity losses between $40^{\circ} \mathrm{S}$ to $40^{\circ} \mathrm{N}$. At latitudes higher than $40^{\circ}$, we found possible gains in biodiversity, regardless of the adaptation scenario and the evolution hypothesis. At these latitudes, for most phytoplankton species, the optimum temperature $\left(T_{\mathrm{opt}}\right)$ is higher than the average environmental temperature $\left(\bar{T}_{S}\right)$. With a fast adaptation scenario, thermal traits follow the thermal environment and $T_{\text {opt }}$ remains above $\bar{T}_{S}$, each thermotype keeps its thermal niche and diversity is not affected. In contrast, thermal traits will not change fast enough in a slow adaptation scenario; $\bar{T}_{S}$ gets closer to $T_{\text {opt }}$, and each thermotype ends up with a fitness that is out of phase with the thermal environment (Supplementary Fig. 18). While these conditions are still favorable for growth, they typically increase the diversity. Finally, for the adaptation scenario where the thermal niche can increase, it gives more chance for a species to adapt faster even for a
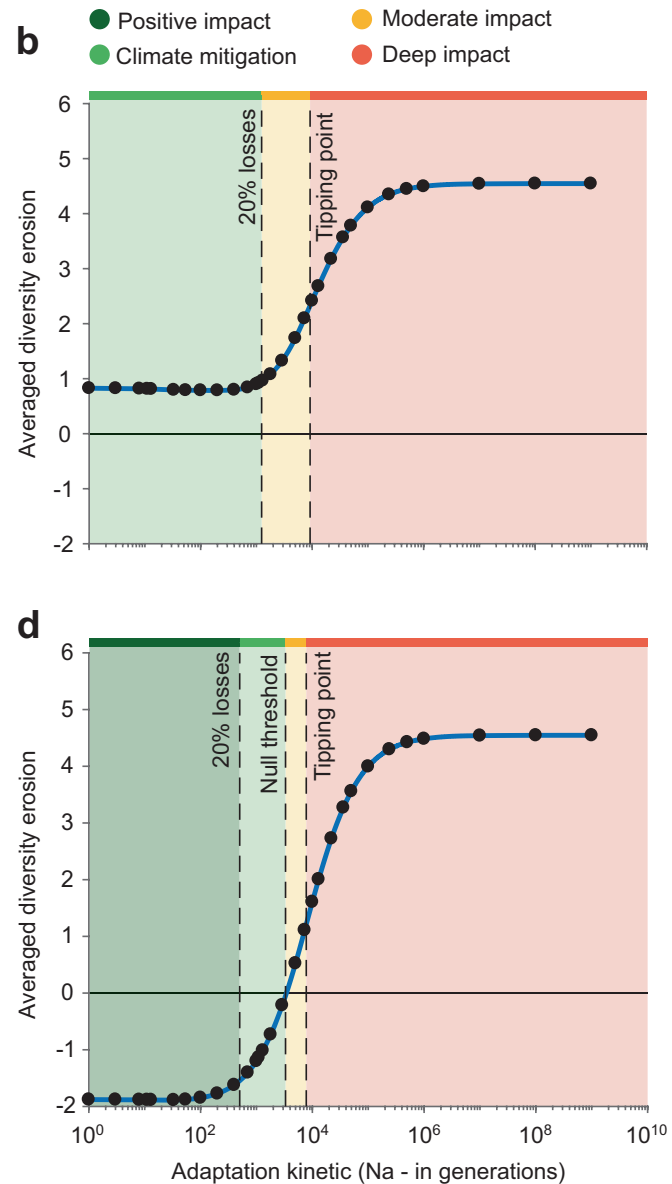

Fig. 5 Micromonas diversity changes in a warming ocean for two evolution hypotheses: a-b Specialist-generalist with constant thermal niche and $\mathbf{c}-\mathbf{d}$ Specialist-generalist with dynamical thermal niche. $\mathbf{a}-\mathbf{b}$ Latitudinal averaged diversity erosion calculated as the difference between diversity in present period (2001-2010) and future (2091-2100). Black line represents the diversity erosion from Thomas et al. [3], red and blue line are the diversity erosion for the fast adaptation scenario $(\mathrm{Na}=100)$ and slow adaptation scenario $(\mathrm{Na}=$
$10^{6}$ ) respectively. Filled area represent the standard deviation to the mean along latitude. c, d Averaged diversity erosion per latitude calculated for different adaptation kinetic (from $\mathrm{Na}=1$ to $\mathrm{Na}=10^{6}$ generations): model results (black circles) and polynomial regression (blue line) fitted. The Tipping point is calculated as the inflexion point for the derivative of blue curve. The $20 \%$ loss point is calculated as $20 \%$ evolution from the lowest erosion scenario $(\mathrm{Na}=1)$ 
higher change in the thermal environment (Supplementary Fig. 19). At latitudes lower than $40^{\circ}$, ocean warming will drive a decrease in phytoplankton diversity, with a mitigation of diversity losses tightly dependent on the adaptation time scale and similar for both hypotheses (Fig. 5a, c). Slow adaptation scenarios lead to an important diversity erosion compared to fast adaptation scenarios, suggesting that the adaptation time scale is a key parameter in the mitigation of diversity loss and matters far more than the strategy of adaptation itself. In areas most vulnerable to diversity erosion (Supplementary Fig. 20 and 21), faster adaptation reduces the average diversity erosion from 4.5 species lost per latitude degree (slow adaptation) to one species lost or even 2 species gained per latitude (fast adaptation, Fig.s 5b, d). Thermal adaptation performed within 200-300 generations might be sufficient to mitigate the impacts of climate change on phytoplankton diversity. In contrast, an adaptation scale beyond $10^{4}$ generations will not counteract the deep impacts of climate change on phytoplankton diversity.

The adaptation time scale of the thermal tolerance of different phytoplankton taxa has been closely related to their respective thermal environments (measured with $T_{\text {opt }}$ or the Net Primary Production) [53, 54, 71, 72]. Phytoplankton taxa that ought to efficiently adapt to temperature are encountered in highly variable thermal environments [71], typically found at latitudes beyond $40^{\circ}$, where we found positive change in future diversity. These regions are also the main areas of $\mathrm{CO}_{2}$ mitigation and carbon export in the ocean $[2,73]$. The deeper alteration of phytoplankton diversity in the tropics might prove less critical for the efficiency of the biological pump at the global scale. Future research should be addressed to understand the impact of microbial diversity on carbon export [74].

\section{Conclusion}

This study describes niche partitioning in the marine picophytoplankton Micromonas. We showed that this genus evolved into different thermotypes that discriminate according to their sensitivity to temperature. Our model predictions were validated by in situ data from the Tara Oceans scientific expedition and suggest that temperature is a robust descriptor of Micromonas distribution at mesoscale and on a yearly basis. The diversity within this genus is highly correlated to the diversity pattern of the whole phytoplankton community. It is crucial to dedicate specific efforts to monitor the evolution of this sentinel genus in order to keep a real-time high fidelity picture of the phytoplankton diversity across the oceans. It is likely that Micromonas genus comprises even more thermotypes. More refined laboratory assessments including more thermotypes, should they exist, would enhance the representation of the global phytoplankton distribution. In particular, new experiments with smaller temperature increments and including more points at low and high temperatures would provide with a much higher resolution in the predicted capabilities and better assessment of $T_{\min }$ and $T_{\max }$.

Although decisive, the ability of phytoplankton to adapt in a warming ocean is the yet uncertain parameter. Adaptation is directly or indirectly affected by a variety of factors such as local nutrient availability, predation, virus lysis, mixing regime, etc. All of them are affected by the local physical dynamics and will also be impacted by global warming. More research is thus required to understand the adaptation mechanisms of this sentinel organism, and especially the adaptive dynamics of the different thermotypes. Such an approach will progressively refine the picture of phytoplankton evolution in a changing ocean with the possibility to more rapidly detect tipping points.

Acknowledgements We thank members of the RCC for their help and work to maintain many phytoplankton strains in culture. We are also grateful to Mridul Thomas for providing his phytoplankton diversity data [3] and to Keith Paarporn for proofreading the text. Finally, we thank the reviewers and editor for their constructive comments on a previous version of this manuscript. This research was funded by the Inria Project Lab Algae in silico and by the ANR funding agency REVIREC (grant no. 12-BSV7-0006-01).

Author contributions $\mathrm{DD}, \mathrm{ACB}, \mathrm{OB}, \mathrm{NS}, \mathrm{AS}$, and SR designed the study. DD, ACB, NS, PG, FRJ carried out the experiments. DD and OB carried out the modeling and statistical analyses. DD provided the display items. AM carried out the phylogenetic and Tara Ocean V9 dataset analysis. CS and DM helped technically. DD, OB, and SR wrote the manuscript with contributions from NS, CS, and $\mathrm{ACB}$.

\section{Compliance with ethical standards}

Conflict of interest The authors declare that they have no conflict of interest.

\section{References}

1. Pachauri RK, Allen MR, Barros VR, Broome J, Cramer W, Christ R, et al. Climate change 2014: synthesis Report. Contribution of working groups I, II and III to the fifth assessment report of the intergovernmental panel on climate change. IPCC; 2014. https://www.springer.com/us/book/9780387981413

2. Sabine CL, Feely RA, Gruber N, Key RM, Lee K, Bullister JL, et al. The oceanic sink for anthropogenic $\mathrm{CO}_{2}$. Science. 2004;305: 367-71.

3. Thomas MK, Kremer CT, Klausmeier CA, Litchman E. A global pattern of thermal adaptation in marine phytoplankton. Science. 2012;338:1085-8.

4. Irwin AJ, Finkel ZV, Muller-Karger FE, Troccoli Ghinaglia L. Phytoplankton adapt to changing ocean environments. PNAS. 2015;112:5762-6.

5. Reid PC, Fisher AC, Lewis-Brown E, Meredith MP, Sparrow M, Andersson AJ, et al. Impacts of the oceans on climate change. Adv Mar Biol. 2009;56:1-150. 
6. Sunagawa S, Coelho LP, Chaffron S, Kultima JR, Labadie K, Salazar G, et al. Structure and function of the global ocean microbiome. Science. 2015;348:1261359.

7. Farrant GK, Doré H, Cornejo-Castillo FM, Partensky F, Ratin M, Ostrowski M, et al. Delineating ecologically significant taxonomic units from global patterns of marine picocyanobacteria. PNAS. 2016;113:E3365-E3374.

8. Rutherford S, D'Hondt S, Prell W. Environmental controls on the geographic distribution of zooplankton diversity. Nature. 1999; 400:749-52.

9. Fuhrman JA. Microbial community structure and its functional implications. Nature. 2009;459:193-9.

10. Tittensor DP, Mora C, Jetz W, Lotze HK, Ricard D, Berghe EV, et al. Global patterns and predictors of marine biodiversity across taxa. Nature. 2010;466:1098-103.

11. Jun Sul W, Oliver TA, Ducklow HW, Amaral-Zettler LA, Sogin ML. Marine bacteria exhibit a bipolar distribution. PNAS. 2013;110:2342-7.

12. Wiens JJ. The niche, biogeography and species interactions. Phylosophical Trans R Soc B Biol Sci. 2011;366:2336-50.

13. Pittera J, Humily F, Thorel M, Grulois D, Garczarek L, Six C. Connecting thermal physiology and latitudinal niche partitioning in marine Synechococcus. ISME J. 2014;8:1221-36.

14. Martiny AC, Ma L, Mouginot C, Chandler JW, Jeremy W, Zinser ER. Interactions between thermal acclimatation, growth rate, and phylogeny influence Prochlorococcus elemental stoichiometry. PLoS ONE. 2016;11:e0168291.

15. Lovejoy C, Vincent WF, Bonilla S, Roy S, Martineau MJ, Terrado $\mathrm{R}$, et al. Distribution, phylogeny, and growth of cold-adapted picoprasinophytes in Arctic seas. J Phycol. 2007;43:78-89.

16. Worden AZ, Not F. Ecology and diversity of picoeukaryotes. In: Gasol JM, Kirchman DL. editors. John Wiley \& Sons. Microbial ecology of the oceans. 2nd ed.; 2008. p. 159-205.

17. Monier A, Sudek S, Fast NM, Worden AZ. Gene invasion in distant eukaryotic lineages: discovery of mutually exclusive genetic elements reveals marine biodiversity. ISME J. 2013; 7:1764-74.

18. Monier A, Comte J, Babin M, Forest A, Matsuoka A, Lovejoy C. Oceanographic structure drives the assembly processes of microbial eukaryotic communities. ISME J. 2015;9:990-1002.

19. Not F, Latasa M, Marie D, Cariou T, Vaulot D, Simon N. A single species, Micromonas pusilla (Prasinophyceae), dominates the eukaryotic picoplankton in the Western English Channel. Appl Environ Microbiol. 2004;70:4064-72.

20. Massana R. Eukaryotic picoplankton in surface oceans. Annu Rev Microbiol. 2011;65:91-110.

21. Guillou L, Eikrem W, Chrétiennot-Dinet MJ, Le Gall F, Massana $\mathrm{R}$, Romari K, et al. Diversity of picoplanktonic prasinophytes assessed by direct nuclear SSU rDNA sequencing of environmental samples and novel isolates retrieved from oceanic and coastal marine ecosystems. Protist. 2004;155:193-214.

22. Slapeta J, Lopez-Garcia P, Moreira D. Global dispersal and ancient cryptic species in the smallest marine eukaryotes. Mol Biol Evol. 2006;23:23-9.

23. Foulon E, Not F, Jalabert F, Cariou T, Massana R, Simon N. Ecological niche partitioning in the picoplanktonic green alga Micromonas pusilla: evidence from environmental surveys using phylogenetic probes. Environ Microbiol. 2008;10:2433-43.

24. Marin B, Melkonian M. Molecular phylogeny and classification of the Mamiellophyceae class. nov. (Chlorophyta) based on sequence comparisons of the nuclear-and plastid-encoded rRNA operons. Protist. 2010;161:304-36.

25. Simon N, Foulon E, Grulois D, Six C, Desdevises Y, Latimier M, et al. Revision of the genus Micromonas Manton et Parke (Chlorophyta, Mamiellophyceae), of the type species M. pusilla (Butcher) Manton \& Parke and of the species M. commoda van
Baren, Bachy and Worden and description of two new species based on the genetic and phenotypic characterization of cultured isolates. Protist. 2017;168:612-35.

26. Johnson ZI, Zinser ER, Coe A, McNulty NP, Woodward EMS, Chisholm SW. Niche partitioning among Prochlorococcus ecotypes along ocean-scale environmental gradients. Science. 2006; 311:1737-40.

27. De Vargas C, Audic S, Henry N, Decelle J, Mahé F, Logares R, et al. Eukaryotic plankton diversity in the sunlit ocean. Science. 2015;348:1261605.

28. Worden AZ, Lee JH, Mock T, Rouzé P, Simmons MP, Aerts AL, et al. Green evolution and dynamic adaptations revealed by genomes of the marine picoeukaryotes Micromonas. Science. 2009;324:268-72.

29. Keller MD, Selvin RC, Claus W, Guillard RRL. Media for the culture of oceanic ultraphytoplankton. J Phycol. 1987;23:633-8.

30. Marie D, Brussaard CPD, Thyrhaug R, Bratbak G, Vaulot D. Enumeration of marine viruses in culture and natural samples by flow cytometry. Appl Environ Microbiol. 1999;65:45-52.

31. Bernard O, Rémond B. Validation of a simple model accounting for light and temperature effect on microalgal growth. Bioresour Technol. 2012;123:520-7.

32. Grimaud GM, Mairet F, Sciandra A, Bernard O. Modeling the temperature effect on the specific growth rate of phytoplankton: a review. Rev Environ Sci Bio/Technol. 2017;16:625-45.

33. Low-Decarie E, Boatman TG, Bennet N, Passfield W, GavalasOlea A, Siegel P, et al. Predictions of response to temperature are contingent on model choice and data quality. Ecol Evol. 2017; $7: 10467-81$.

34. Norberg J. Biodiversity and ecosystem functioning: a complex adaptive systems approach. Limnol Oceanogr. 2004;49:1269-77.

35. Boatman TG, Lawson T, Geider RJ. A key marine diazotroph in a changing ocean: The interacting effects of temperature, $\mathrm{CO} 2$ and light on the growth of Trichodesmium erythraeum IMS101. PLoS ONE. 2017;12:e0168796.

36. Katoh K, Standley DM. MAFFT multiple sequence alignment software version 7: improvements in performance and usability. Mol Biol Evol. 2013;30:772-80.

37. Stamatakis A. RaxML version 8: a tool for phylogenetic analysis and post-analysis of large phylogenies. Bioinformatics. 2014;30: 1312-3.

38. Darriba D, Taboada GL, Doallo R, Posada D. jModelTest 2: more models, new heuristics and parallel computing. Nat Methods. 2012;9:772-772.

39. Bouckaert R, Heled J, Kuhnert D, Vaughan T, Wu CH, Xie D, et al. BEAST 2: a software platform for Bayesian evolutionary analysis. PLoS Comput Biol. 2014;10:e1003537.

40. Letunic I, Bork P. Interactive tree of life v2: online annotation and display of phylogenetic trees made easy. Nucleic Acids Res. 2011;39:W475-8.

41. Berger SA, Stamatakis A. Aligning short reads to reference alignments and trees. Bioinformatics. 2012;27:2068-75.

42. Oksanen J, Kindt R, Legendre P, Minchin PR, O'hara RB, Simpson GL, et al. Vegan: community ecology package. R package version 2.4-0. 2016. https://CRAN.R-project.org/packa ge $=$ vegan .

43. Kruskal JB. Multidimensional scaling by optimizing goodness of fit to a nonmetric hypothesis. Psychometrika. 1964;29:1-27.

44. Eppley RW. Temperature and phytoplankton growth in the sea. Fish Bull. 1972;70:1063-85.

45. Grimaud GM. Modelling the temperature effect on phytoplankton: from acclimation to adaptation. Doctoral dissertation, University Nice Sophia Antipolis; 2016.

46. Pesant S, Not F, Picheral M, Kandels-Lewis S, Le Bescot N, Gorsky G, et al. Open science resources for the discovery and analysis of Tara Oceans data. Sci Data. 2015;2:150023. 
47. Mahé F, Rognes T, Quince C, de Vargas C, Dunthorn M. Swarm: robust and fast clustering method for amplicon-based studies. PeerJ. 2014;2:e593.

48. Guillou L, Bachar D, Audic S, Bass D, Berney C, Bittner L, et al. The Protist Ribosomal Reference database (PR2): a catalog of unicellular eukaryote small sub-unit rRNA sequences with curated taxonomy. Nucleic Acids Res. 2012;41:D597-D604.

49. Wickham H. ggplot2: elegant graphics for data analysis. Springer; 2016. https://www.springer.com/us/book/9780387981413link

50. Griffies SM, Gnanadesikan AWDK, Dixon KW, Dunne JP, Gerdes R, Harrison MJ, et al. Formulation of an ocean model for global climate simulations. Ocean Sci. 2005;1:45-79.

51. Delworth T, Broccoli AJ, Rosati A, Stouffer RJ, Balaji V, Beesley JA, et al. GFDL's CM2 global coupled climate models. Part I: Formulation and simulation characteristics. J Clim. 2006;19:643-74.

52. Nakicenovic N, Alcamo J, Grubler A, Riahi K, Roehrl RA, Rogner HH, et al. Special report on emissions scenarios (SRES), a special report of Working Group III of the intergovernmental panel on climate change. In: Nakicenovic N, Swart R, editors. Cambridge, UK: Cambridge University Press; 2000.

53. Padfield D, Yvon-Durocher G, Buckling A, Jennings S, YvonDurocher G. Rapid evolution of metabolic traits explains thermal adaptation in phytoplankton. Ecol Lett. 2016;19:133-42.

54. Bonnefond H, Grimaud G, Rumin J, Bougaran G, Talec A, Gachelin $\mathrm{M}$, et al. Continuous selection pressure to improve temperature acclimation of Tisochrysis lutea. PLoS ONE. 2017;12:e0183547.

55. Boyd PW, Rynearson TA, Armstrong EA, Fu F, Hayashi K, Hu Z, et al. Marine phytoplankton temperature versus growth responses from polar to tropical waters-outcome of a scientific community wide study. PLoS ONE. 2013;8:e63091.

56. Raven JA, Geider RJ. Temperature and Algal Growth. New Phytol. 1988;110:441-61.

57. Serra-Maia R, Bernard O, Gonçalves A, Bensalem S, Lopes F. Influence of temperature on Chlorella vulgaris growth and mortality rates in a photobiore actor. Algal Res. 2016;18: 352-9.

58. Salvucci ME, Crafts-Brandner SJ. Inhibition of photosynthesis by heat stress: the activation state of Rubisco as a limiting factor in photosynthesis. Physiol Plant. 2004;120:179-86.

59. Rokka A, Aro EM, Herrmann RG, Andersson B, Vener AV. Dephosphorylation of photosystem II reaction center proteins in plant photosynthetic embranes as an immediate response to abrupt elevation of temperature. Plant Physiol. 2016;123:1525-36.

60. Los DA, Murata N. Membrane fluidity and its roles in the perception of environmental signals. Biochim Et Biophys Acta. 2004; 1666:142-57.

61. Cuvelier ML, Allenc AE, Monier A, McCrow JP, Messie M, Tringed SG, et al. Targeted metagenomics and ecology of globally important uncultured eukaryotic phytoplankton. Proc Natl Acad Sci. 2010;107:14679-84.

62. Monier A, Worden AZ, Richards TA. Phylogenetic diversity and biogeography of the Mamiellophyceae lineage of eukaryotic phytoplankton across the oceans. Environ Microbiol Rep. 2016;8: 461-9.

63. Demir-Hilton E, Sudek S, Cuvelier ML, Gentemann CL, Zehr JP, Worden AZ. Global distribution patterns of distinct clades of the photosynthetic picoeukaryote Ostreococcus. ISME J. 2011;5: 1095-107.

64. Chen B. Patterns of thermal limits of phytoplankton. J Plankton Res. 2015;37:285-92.

65. Rosso L, Lobry JR, Flandrois JP. An unexpected correlation between cardinal temperatures of microbial growth highlighted by a new model. J Theor Biol. 1993;162:447-63.

66. Baudoux AC, Lebredonchel H, Dehmer H, Latimier M, Edern R, Rigaut-Jalabert F, et al. Interplay between the genetic clades of Micromonas and their viruses in the Western English Channel. Environ Microbiol Rep. 2015;7:765-73.

67. Lennon JT, Jones SE. Microbial seed banks: the ecological and evolutionary implications of dormancy. Nat Rev Microbiol. 2011;9:119-30.

68. Botebol H, Lelandais G, Six C, Lesuisse E, Meng A, Bittner L, et al. Acclimatation of a low iron adapted Ostreococcus strain to iron limitation through cell biomass lowering. Sci Report. 2017;7:327.

69. Grimaud GM, Le Guennec V, Ayata SD, Mairet F, Sciandra A, Bernard O. Modelling the effect of temperature on phytoplankton growth across the global ocean. IFAC-Pap. 2015;48: 228-33.

70. Stinchcombe JR, Function-valued Traits Working Group, Kirkpatrick M. Genetics and evolution of function-valued traits: understanding environmentally responsive phenotypes. Trends Ecol Evol. 2012;27:637-47.

71. Huertas IE, Rouco M, Lopez-Radas V, Costas E. Warming will affect phytoplankton differently: evidence through a mechanistic approach. Proc R Soc B. 2011;278:3534-43.

72. Schaum CE, Barton S, Bestion E, Buckling A, Garcia-Carreras B, Lopez $\mathrm{P}$, et al. Adaptation of phytoplankton to a decade of experimental warming linked to increased photosynthesis. Nat Ecol Evol. 2017;1:0094.

73. Lutz MJ, Caldeira K, Dunbar RB, Behrenfeld MJ. Seasonal rhythms of net primary production and particulate organic carbon flux to depth describe the efficiency of biological pump in the global ocean. J Geophys Res. 2007;112:C10.

74. Guidi L, Chaffron S, Bittner L, Eveillard D, Larhlimi A, Roux S, et al. Plankton networks driving carbon export in the oligotrophic ocean. Nature. 2016;532:465. 